\title{
Performance Enhancement of Joint Adaptive Modulation, Coding and Power Control Using Cochannel-Interferer Assistance and Channel Reallocation*
}

\author{
Mohamed H. Ahmed, Halim Yanikomeroglu, David Falconer, and Samy Mahmoud \\ BCWS Centre, Dept. of Systems and Computer Eng., Carleton University, \\ 1125 Colonel By Dr., Ottawa, Canada
}

\begin{abstract}
This paper proposes a joint adaptive rate selection and power control algorithm for broadband TDMA/TDM wireless networks. The proposed algorithm is a modified, enhanced, and more robust version of the Selective Power Control with Active Link Protection (SPC-ALP) algorithm proposed for adaptive transmission rate and power control in CDMA networks [1]. Unlike SPC-ALP, the proposed algorithm uses adaptive coding and modulation (instead of variable spreading gain) for transmission rate control. In addition, the proposed algorithm is different in two aspects; the first one is the inclusion of the cochannel interferer assistance mode, while the second one is the use of the signal quality as a criterion for user removal. Results show that the proposed algorithm outperforms SPC-ALP in terms of the net throughput and signal quality measures such as the outage probability and frame error rate. Channel reallocation is also studied and found to be very effective in enhancing the system performance particularly at low to medium loading.
\end{abstract}

\section{INTRODUCTION}

There is a growing demand for broadband access due to the increasing popularity of the Internet and multimedia services. There are various competing technologies for broadband access networks, namely, xDSL, optical fibre, cable modems, and broadband wireless access. The latter has attracted more attention because of its fast and easy deployment, low installation cost, and scalable roll out plans. However, radio resources must be efficiently managed to provide high transmission rate $(10 \mathrm{Mb} / \mathrm{s}$ or more) with a good quality of service (QoS) despite the wireless propagation impairments such as shadowing, multipath fading, and interference.

Adaptive resource allocation is one of the most effective techniques for QoS provisioning in wireless networks. Adaptive power control has been employed in $2 \mathrm{G}$ and $3 \mathrm{G}$ cellular systems for combating channel impairments and enhancing the signal quality. Power control and transmission rate allocation is a constrained optimization problem with an objective of maximizing the throughput subject to some constraints such as maximum power level and/or maximum outage probability. This problem has been addressed in [2] for CDMA systems. The algorithm given in [2], Selective Power Control (SPC), has been proposed as an efficient technique for power control and transmission rate selection. In CDMA systems, the transmission rate is adapted by changing the

\footnotetext{
* This work is supported by the National Capital Institute of

Telecommunication (NCIT), Ottawa, Ontario, Canada.
}

spreading gain. Although SPC is effective in maximizing the system throughput, it doesn't protect the active users from the signal quality degradation due to the arrival of new users or large power step-up from users seeking higher transmission rate. Kim et al. [1] have proposed a more robust algorithm by combining SPC technique with an admission and signal quality control scheme called Active Link Protection (ALP) [3]. With ALP, power step-up of both new users and those seeking higher transmission rate is limited by a small step size value $(\delta>1)$.

Adaptive coding and modulation techniques have been proposed for maximizing the system throughput by adapting the modulation level and the coding rate to the channel conditions, e.g. [4-6]. Combining adaptive coding and modulation techniques with power control in one joint algorithm in broadband TDMA/TDM wireless access networks is the main topic of this paper. We have adopted the same approach of SPC-ALP for the transmission rate selection and power control, but with the use of adaptive coding and modulation instead of variable spreading gain. The proposed algorithm has two main modifications; the first one is the inclusion of cochannel-interferer assistance, while the second is the signal quality-based removal. The performance of the proposed algorithm has been analyzed and compared with that of SPC-ALP in broadband fixed wireless network environment. Throughout the rest of this paper, the proposed algorithm is denoted SPC-ALP with Assistance and Signal Quality-based Removal (SCP-ALP-ASQR). We have also studied the impact of channel reallocation on the performance of both algorithms (SPC-ALP and SPC-ALP-ASQR). The rest of this paper is organized as follows: In the next section, the system model and main assumptions are presented. The proposed algorithm is explained in section III. Then, the results are provided in section IV. Finally, the conclusions and future work are given in section $\mathrm{V}$.

\section{SYSTEM MODEL}

A hexagonal cellular structure is considered in this study. Each cell is divided into 6 sectors. Thus, an ideal beam pattern with a $60^{\circ}$-beamwidth, main lobe gain of $20 \mathrm{~dB}$, and side lobe gain of $0 \mathrm{~dB}$ is used at the basestation (BS). Similarly, Subscriber Stations (SSs) have directional antennas with a $60^{\circ}$ - 
beamwidth, main lobe gain of $15 \mathrm{~dB}$, and side lobe gain of 0 $\mathrm{dB}$. While the BS antenna beams are fixed, it is assumed that the antenna beams at the SSs are electronically steered to point at the direction of serving BSs.

The channel model consists of an exponential path loss model with an exponent $(n)$ of 3.5 , lognormal shadowing with a standard deviation $(\sigma)$ of 8 , and flat Rayleigh fading. Shadowing samples are spatially correlated with 0.5 correlation coefficient for $1 \mathrm{~m}$ displacement. Rayleigh fading samples are temporally correlated using rounded (bell-shaped) Doppler spectrum with a $3-\mathrm{dB}$ frequency of $2 \mathrm{~Hz}$ [7]. The Rayleigh fading samples of a user from different BSs are mutually independent.

A frequency reuse plan of $1 / 6$ is employed such that the total spectrum is divided into 6 equal sub-bands allocated to the 6 sectors of each cell. The whole spectrum is reused every cell. This tight frequency reuse plan can be used since directional beams are employed at both BS and SS. A total bandwidth of $36 \mathrm{MHz}$ is partitioned into 6 equal sub bands so that each sector is allocated a $6 \mathrm{MHz}$ channel.

Time is divided into frames with $10 \mathrm{~ms}$ frame duration and 8 slots per frame in a TDMA fashion. 11 combinations of coding-modulation levels using Bit-Interleaved Coded Modulation (BICM) [8] are utilized to adapt the transmission rate to the channel conditions and interference variation. TableI lists these 11 coding-modulation combinations associated with their spectral efficiency and signal to interference and noise ratio (SINR) requirements at $10^{-6}$ BER. Power level, coding rate and modulation level are updated once per frame.

This work focuses on the downlink performance since it is the limiting factor in many multimedia services that show asymmetrical behavior. Fixed users are considered here. However, the proposed algorithm can be used by nomadic/mobile users. The transmitted power has a $20 \mathrm{~dB}$ dynamic range and a maximum value $\left(p_{\max }\right)$ of $30 \mathrm{dBm}$. A continuous transmission is assumed, which corresponds to stream-mode services or aggregate traffic of several sources in a LAN. It is assumed that users are uniformly distributed and are assigned to the best serving BS.

TABLE I
SINR OF DIFFERENT CODING- MODULATION LEVELS

\begin{tabular}{|c|c|c|c|}
\hline $\begin{array}{c}\text { Coding- } \\
\text { modulation } \\
\text { level }(k)\end{array}$ & $\begin{array}{c}\text { Coding rate \& } \\
\text { Modulation level } \\
\text { combination }\end{array}$ & $\begin{array}{c}\text { Spectral } \\
\text { Efficiency } \\
(\mathrm{b} / \mathrm{s} / \mathrm{Hz})\end{array}$ & $\begin{array}{c}\text { SINR } \text { at } \\
10^{-6} \mathrm{BER} \\
\left(\gamma^{k}\right) \mathrm{dB}\end{array}$ \\
\hline 1 & $1 / 2$ \& QPSK & 1.00 & 4.65 \\
\hline 2 & $2 / 3$ \& QPSK & 1.33 & 6.49 \\
\hline 3 & $3 / 4$ \& QPSK & 1.50 & 7.45 \\
\hline 4 & $7 / 8$ \& QPSK & 1.75 & 9.05 \\
\hline 5 & $1 / 2 \&$ 16-QAM & 2.00 & 10.93 \\
\hline 6 & 2/3 \& 16-QAM & 2.66 & 12.71 \\
\hline 7 & $3 / 4 \&$ 16-QAM & 3.00 & 14.02 \\
\hline 8 & $7 / 8 \&$ 16-QAM & 3.50 & 15.74 \\
\hline 9 & 2/3 \& 64-QAM & 4.00 & 18.50 \\
\hline 10 & $3 / 4 \&$ 64-QAM & 4.50 & 19.88 \\
\hline 11 & $7 / 8 \&$ 64-QAM & 5.25 & 21.94
\end{tabular}

\section{PROPOSED ALGORITHM}

As shown in Fig. 1, SPC-ALP algorithm defines three modes for users: Passive, Standard, and Transition. In the Passive mode, users do not transmit so the power level is zero. In the Standard mode, users update their power according to (1) [1] provided that the transmission rate is not increasing.

$$
p(n+1)=\max _{k}\left\{\frac{\delta p(n) \gamma^{k}}{\gamma(n)} \chi\left(\frac{\delta p(n) \gamma^{k}}{\gamma(n)}<p_{\max }\right)\right\}
$$

where $p(n)$ is the power level at iteration $n, \gamma(n)$ is the SINR of the coding-modulation level $(k), \gamma^{k}$ is the target SINR of coding-modulation level $(k)$, and $\chi$ is the indicator function.

The transition mode is utilized by new users trying to join the set of active users or active users seeking higher transmission rates. Power is updated in the transition mode as follows [1]

$$
p(n+1)=\delta p(n)
$$

Users who fail in achieving the minimum transmission rate are switched to the Passive mode with probability $p_{1}$, while users in the Passive mode are switched to the Transition mode with probability $p_{2}$. In SPC-ALP, both $p_{1}$ and $p_{2}$ are constant and do not depend on the signal quality. The main objectives of this work are the following:

1-Extending the scope of SPC-ALP algorithm (proposed for rate selection and power control in CDMA systems) to TDMA/TDM systems by controlling the transmission rate using adaptive coding and modulation.

2-Enhancing the signal quality performance by including the assistance mode and the signal quality-based removal policy. 3-Analyzing the algorithm under realistic and dynamic conditions rather than the static condition assumed in [1].

4-Studying the impact of channel reallocation on the performance of SPC-ALP and the proposed algorithm (SPCALP-ASQR).

As shown in Fig. 2, the proposed algorithm (SPC-ALPASQR) has a fourth mode, Assistant mode, in addition to the other three modes proposed in SPC-ALP. When a user is found to be unable to achieve the signal to interference ratio of the minimum transmission rate (lowest coding-modulation level) even with the highest power level, it is switched to the Assistance mode rather than the Passive mode. In this mode, the BS of the assisted user sends a request to the BSs of the cochannel interferers in the surrounding cells to lower their transmission power by moving to a lower coding-modulation level. The assisted users keep sending using the lowest codingmodulation level $(k=1)$ with the maximum power level $\left(p_{\max }\right)$. By doing so, the interference level at the assisted user will be significantly less. The assisting users update their power, modulation and coding combination as follows 


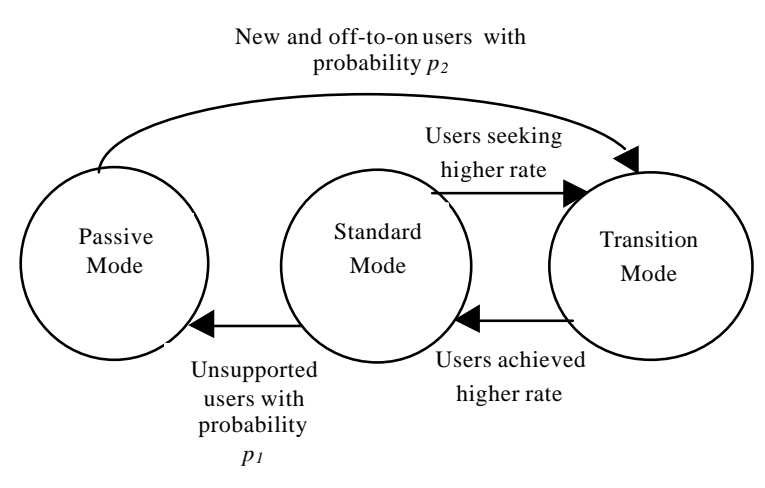

Fig. 1.Transmission modes of the SPC-ALP algorithm

$$
p(n+1)=p(n)-\max (\text { offset }, \alpha p(n)),
$$

where offset is equal to the difference between the minimum SINR and the achieved SINR at iteration $n$ for the assisted user, and $\alpha(<1)$ is a constant used to control the power reduction of assisting users to protect them from sacrificing their signal quality while trying to assist other users. For instance, if $\alpha=0.33$ this ensures that the power reduction for the assisting users does not exceed $33 \%$ of the current power level. Accordingly, the coding-modulation level $(k)$ of the assisting users is chosen by assuming that the SINR will drop by the same ratio of the power reduction. This assumption is pessimistic and gives a lower bound for the SINR of the assisting user. This is because the reduction in the signal power of the assisting users is associated with some reduction in the interference level since other cocochannel interferers except the assisted users are going to lower their transmit power. Thus, $k$ is given by

$$
k=\max \left\{i \chi\left([\gamma(n)-? p]<\delta \gamma^{i}\right)\right\}
$$

where $\Delta p$ is the power reduction. If the assisted user is still unable to achieve the minimum SINR target even after the assistance, then it will be switched to the Passive mode with probability $f_{l}$. Users are switched from the Passive mode to the transition mode with probability $f_{2}$. Unlike $p_{1}$ and $p_{2}$ in SPCALP, both $f_{1}$ and $f_{2}$ are functions of the SINR as follows

$$
\begin{gathered}
f_{1}=\max \left[1, \beta_{1}\left\{\gamma^{l}-\gamma(n)\right\}\right], \\
f_{2}=\max \left[1, \beta_{2}\left\{\gamma_{\text {est }}(n)-\gamma^{l}\right\}\right],
\end{gathered}
$$

where $\beta_{1}$ and $\beta_{2}$ are constants and $\gamma_{\text {est }}(n)$ is the estimated SINR of the temporarily removed users. In $(6), \gamma_{e s t}(n)$ is used since users in the passive mode are off and their actual SINR is zero.

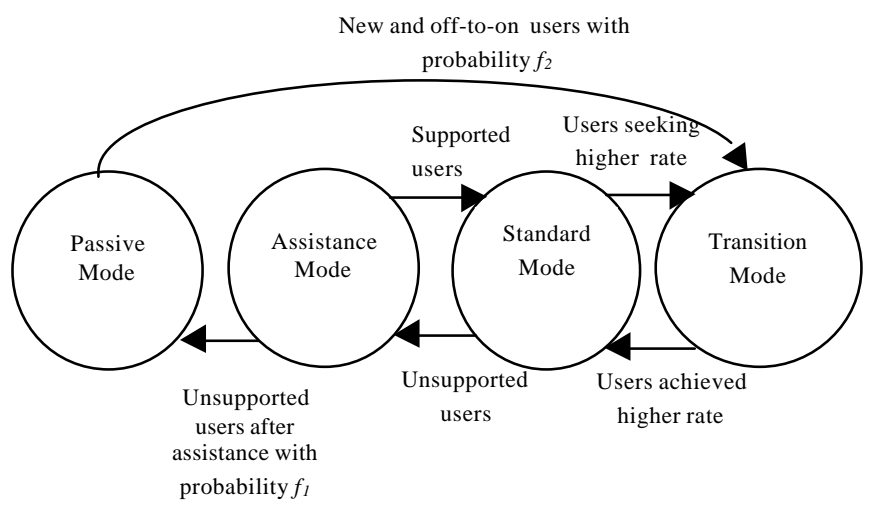

Fig. 2.Transmission modes of the SPC-ALP-ASQR algorithm

It is worth noting that (6) is used to determine the probability of turning removed (off) user to on. Hence, (6) is applied only for removed users who have $\gamma_{e s t}(n)>\gamma^{l} \cdot \gamma_{e s t}(n)$ is given by

$$
\gamma_{e s t}(n)=\frac{p_{m} g}{N+\sum_{j} I_{j}}
$$

where $g$ is the channel gain between the removed user and its BS (including the antenna gain, path loss, shadowing and fading), $N$ is the average noise power, and $I_{j}$ is the interference from the cochannel interferer in cell $j$. Since the removed user does not transmit, the maximum transmit power $\left(p_{\max }\right)$ is used in the estimation of the signal to interference and noise ratio $\gamma_{e s t}(n)$ to ensure that the removed user can achieve the minimum SINR with a feasible power ( $p_{\max }$ in the worst case).

Channel reallocation is employed to enhance the system performance. Users in outage (fail in achieving the minimum coding-modulation level even with the maximum power) are moved to new channels instead of switching them to the passive mode. Idle channels are sorted according to the interference level. When a user is in outage (even after the assistance of the cochannel interferers in case of the SPC-ALPASQR) is assigned the new channel having the lowest interference. If there is no available channel users in outage are switched to the passive mode.

\section{RESULTS}

The system performance of both algorithms (SPC-ALP and SPC-ALP-ASQR) has been analyzed by computer simulation using a broadband wireless network simulation tool [9]. 9 cells with a wraparound grid are used in the simulation. $\beta_{1}$ and $\beta_{2}$ are chosen experimentally to be equal to 0.7 and 0.5 respectively. In order to analyze the system performance, the following performance metrics are utilized.

1-The average total throughput per $\mathrm{Hz}$ per user is defined as the average coding-modulation spectral efficiency of all users 
(active and removed), in other words, it is the average transmission rate of all users divided by the channel bandwidth. More importantly as a performance measure is the net throughput per $\mathrm{Hz}$ per user since it evaluates the total useful throughput. Hence, it is defined as the total throughput (per user per $\mathrm{Hz}$ ) after excluding the erroneous frames.

2-Outage probability $\left(P_{\text {out }}\right)$ is defined as

$$
P_{\text {out }}=P\left\{p(n+1)>p_{\max }\right\}, k=1 .
$$

$P_{\text {out }}$ is the probability that a BS is not able to find a feasible power allocation to achieve the minimum SINR target.

3-Frame Error Rate $(F E R)$ is defined as the probability that a user is allocated a certain power level, coding-modulation level, but the achieved SINR turned out to be less than the corresponding target SINR. Hence, FER is given by

$$
F E R=P\left\{\gamma(n)<\gamma^{k}\right\} .
$$

Figs. 3-6 plot the four performance measure discussed above versus the system loading defined as the total number of users divided by the number of channels for both algorithms (SPCALP and SPC-ALP-ASQR).

The total throughput per user per $\mathrm{Hz}$ is depicted in Fig.3. It is apparent that the channel reallocation enhances the total throughput especially with SPC-ALP. This is because channel reallocation reduces the number of user switched to the passive modes. The total throughput of the proposed algorithm is less than that of SPC-ALP since the assistance mode of the proposed algorithm reduces the transmission rate of some users. It is also worth noting that the throughput enhancement due to the channel reallocation is decreasing with the loading increasing since the availability of good channels (with low interference level) decreases with the system loading increasing.

Fig. 4 shows the net throughput per user per Hz. The channel reallocation enhances the net throughput of SPC-ALP by $25-35 \%$ and from $0 \%$ to $7 \%$ with SPC-ALP-ASQR. Without channel reallocation, the net throughput of SPC-ALPASQR is higher than that of SPC-ALP by $20 \%$. With channel reallocation, the net throughput of both algorithms is almost the same at light ${ }^{*}$ loading and slightly better with SPC-ALP at medium to heavy loading.

The outage probability is plotted in Fig. 5. The proposed algorithm reduces the outage probability significantly for both cases (with and without channel reallocation). Without channel reallocation, SPC-ALP-ASQR reduces the outage probability by $70-80 \%$ compared with SPC-ALP. With channel reallocation, both algorithms perform well (almost no outage) at light loading. At medium to heavy loading, the reduction in the outage probability of SPC-ALP-ASQR ranges from $50 \%$ to $70 \%$. It is evident that a significant reduction of the outage

* Light, medium and heavy loading are defined as loading ratios of less than $30 \%$, 30\%$70 \%$, and higher than $70 \%$ respectively. probability is achievable using channel reallocation especially for SPC-ALP.

Finally, the Frame Error Rate (FER) is depicted in Fig. 6. Without channel reallocation, SPC-ALP-ASQR can decrease the FER by $60 \%$ compared with that of SPC-ALP. With channel reallocation, SPC-ALP-ASQR still outperforms SPCALP but with a less reduction ratio. Although channel reallocation reduces the FER by $25-50 \%$ for SPC-ALP, channel reallocation causes a slight increase in FER for SPCALP-ASQR particularly at medium to heavy loading.

\section{CONCLUSIONS}

A joint adaptive transmission rate selection and power control algorithm (SPC-ALP-ASQR) has been developed for TDM/TDMA broadband wireless networks by modifying and enhancing SPC-ALP algorithm using adaptive coding and modulation, cochannel-interferer assistance, and signal quality removal. Also, channel reallocation has been investigated. It is shown that SPC-ALP with channel reallocation can achieve the highest throughput. However, the proposed algorithm (SPCALP-ASQR) is more robust and outperforms SPC-ALP in terms of the outage probability and frame error rate at the expense of a slight reduction of the net throughput. It is also shown that channel reallocation is less effective with SPCALP-ASQR than with SPC-ALP particularly at high loading values.

\section{ACKNOWLEDGMENT}

The authors would like to thank Dr. S. Ariyavisitakul for providing us with the SINR values of different codingmodulation levels.

\section{REFERENCES}

[1] R. Jantti and S. Kim, "Selective power control with active link protection for combined rate and power management," Proc. IEEE Vehicular Technology Conference Spring, pp. 1960-1964, 2000.

[2] S. Kim, Z. Roseberg, and J. Zander, "Combined power control and transmission rate selection in cellular networks," Proc. IEEE Vehicular Technology Conference Fall, pp. 1653-1657, 1999.

[3] N. Bambos, S. Chen, and G. Pottie, "Radio link admission algorithms for wireless networks with power control and active link protection," Proc. IEEE INFOCOM, pp. 97-104, 1995.

[4] K. Balachandran, S. Kadaba and S. Nanda, "Channel quality estimation and rate adaptation for cellular mobile radio," IEEE JSAC, vol. 17, no. 7, pp. 1244-1256, July 1999.

[5] T. Ue, S. Sampei, N. Morinaga, and K. Hamaguchi, "Symbol rate and modulation level-controlled adaptive modulation TDMA/TDD Systems for high-bit-rate wireless data transmission," IEEE Trans. on Vehicular Technology, vol. 47, no. 4, pp. 1134-1147, Nov. 1998.

[6] E. Armanious, D. Falconer, and H. Yanikomeroglu, "Adaptive modulation, adaptive coding, and power control for fixed cellular broadband wireless systems," Submitted to IEEE WCNC 2003.

[7] D. Baum, "Simulating the SUI channel models," IEEE 802.16.3c-01/53, April 2001.

[8] G. Cair, G. Taricco, and E. Biglieri, "Bit-interleaved coded modulation," IEEE Trans. on Info. Theory, vol. 44, no. 3, pp. 927-946, May 1998.

[9] M. Ahmed, H. Yanikomeroglu, Samy Mahmoud, and David Falconer "A simulation testbed for radio resource management in broadband wireless access networks," Proc. of the $21^{s t}$ Biennial Symposium on Communications 2002, Kingston, Canada, pp. 41-45, June 2002. 


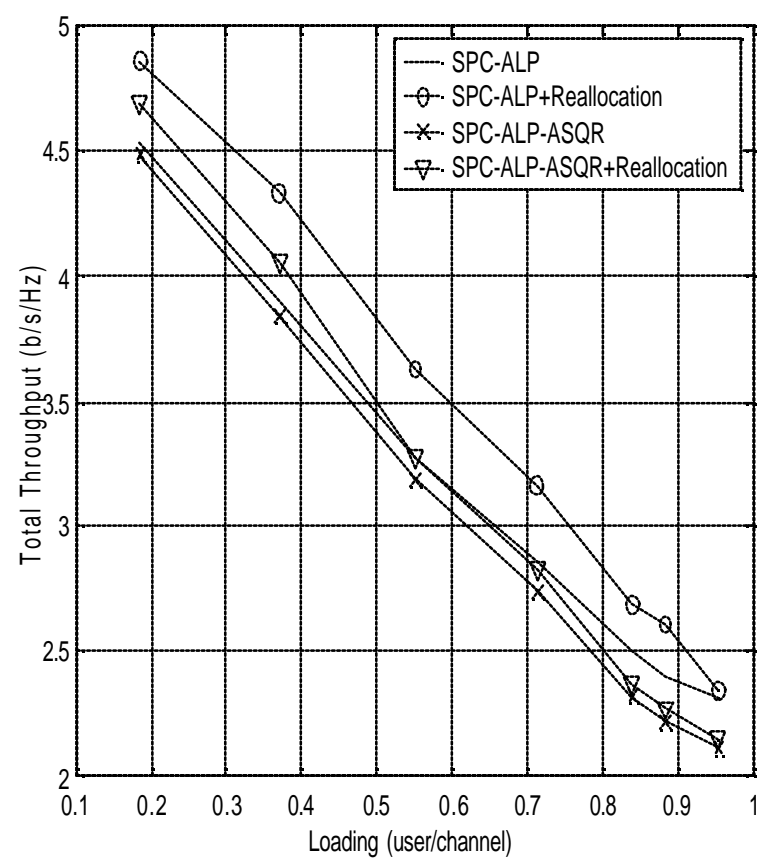

Fig.3. Total Throughput vs. System Loading

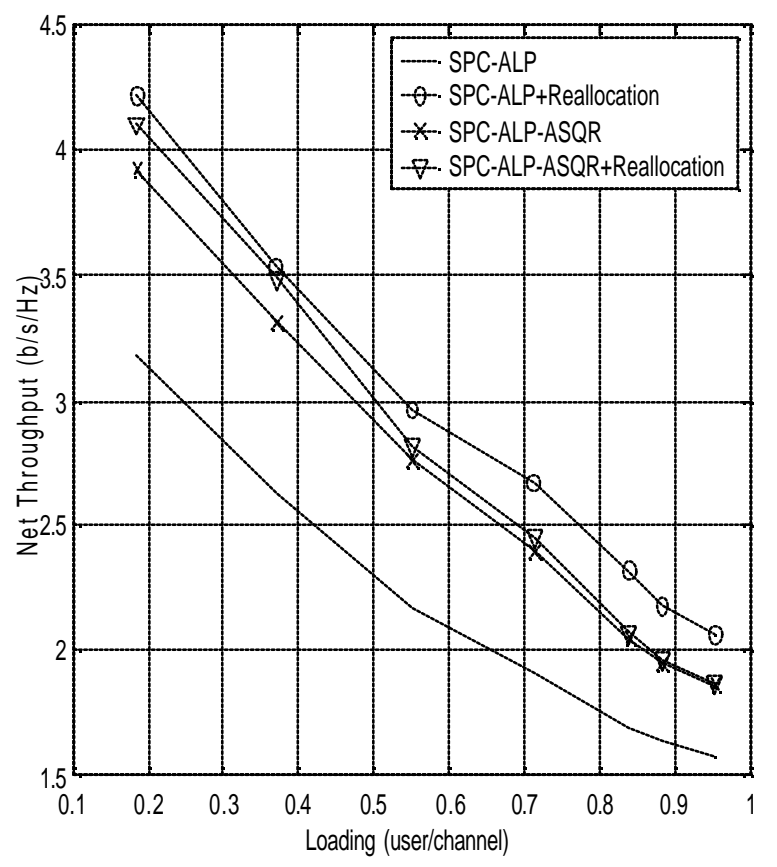

Fig.4. Net throughput vs. System Loading

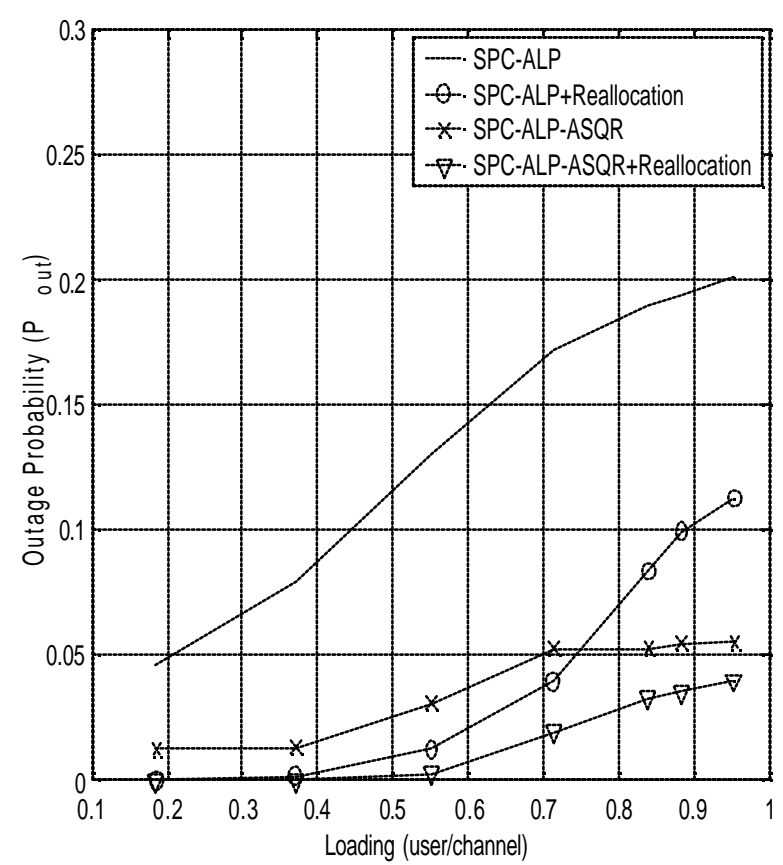

Fig.5. Outage Probability vs. System Loading

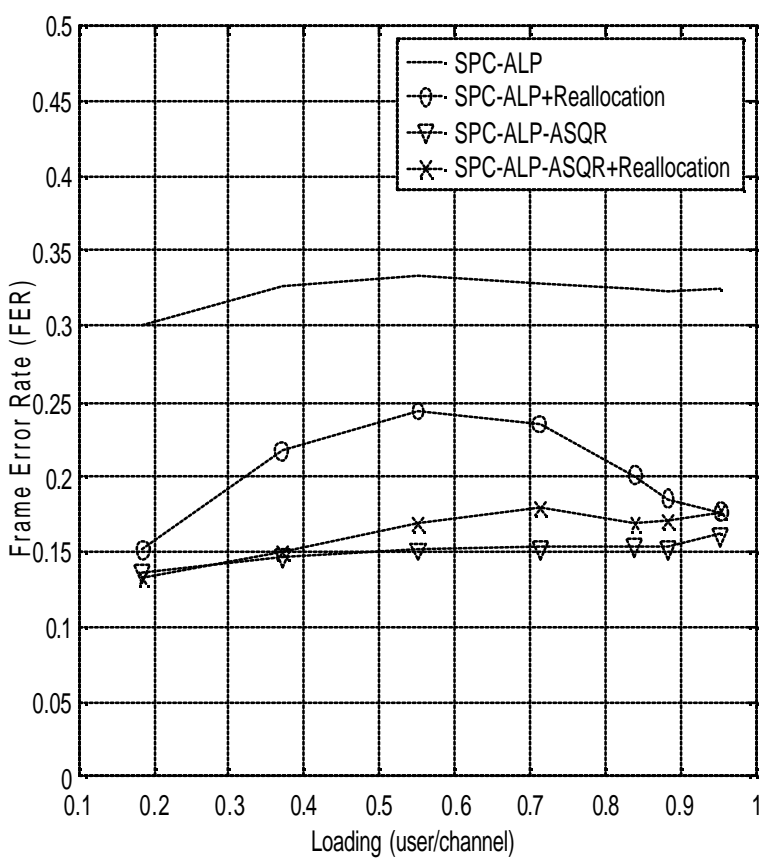

Fig.6. Frame Error Rate vs. System Loading 